\title{
CRISPR/Cas12a-Based Assay for the Rapid and High-Sensitivity Detection of Streptococcus Agalactiae Colonization in Pregnant Women with Premature Rupture of Membrane
}

\section{Donghong Yu}

Fujian Obstetrics and Gynecology Hospital

\section{Bin Liang}

Fujian Maternity and Child Health Hospital, Affiliated Hospital of Fujian Medical University

\section{Haipo Xu}

Mengchao Hepatobiliary Hospital of Fujian Medical University

\section{Zhoujie Ye}

Fujian Obstetrics and Gynecology Hospital

\section{Zhihui Wu}

Fujian Maternity and Child Health Hospital, Affiliated Hospital of Fujian Medical University

Xinrui Wang ( $\square$ wanxiru@sjtu.edu.cn )

Fujian Maternity and Child Health Hospital, Affiliated Hospital of Fujian Medical University

\section{Research Article}

Keywords: Streptococcus agalactiae, Group B Streptococcus, CRISPR-Cas12a, Recombinase polymerase amplification, Intrapartum screening, Premature rupture of membrane

Posted Date: December 13th, 2021

DOI: https://doi.org/10.21203/rs.3.rs-1080306/v1

License: (c) (i) This work is licensed under a Creative Commons Attribution 4.0 International License. Read Full License 


\section{Abstract \\ Background}

Streptococcus agalactiae or group B Streptococcus (GBS) is a leading infectious cause of neonatal morbidity and mortality. This study aims to establish a novel method, termed as the CRISPR-GBS assay, for the rapid and sensitive detection of GBS that is based on the clustered regularly interspaced short palindromic repeats (CRISPR) and CRISPR-associated protein (Cas) system.

\section{Results}

The CRISPR-GBS assay detected GBS in the samples within $35 \mathrm{~min}$. The limit of detection was as low as 5 copies/ $\mu \mathrm{L}$ and showed no cross-reactivity with other microorganisms. The clinical performance was assessed using vaginal or cervical swab samples that were collected from 179 pregnant women with premature rupture of membrane. Compared with the culture-based matrix-assisted laser desorption ionization time-of-flight mass spectrometry method, the CRISPR-GBS assay demonstrated a sensitivity of $96.64 \%(144 / 149,95 \%$ confidence interval $[\mathrm{Cl}]=92.39-98.56 \%)$ and a specificity of $100 \%(30 / 30,95 \% \mathrm{Cl}$ $=88.65-100 \%)$. It also had a high concordance rate of $98.88 \%$ with the real-time fluorescence polymerase chain reaction assay.

\section{Conclusions}

The CRISPR-GBS assay can be used for rapid and high-sensitivity detection of GBS in a simple and costefficient manner; thus, it offers a novel method for intrapartum screening.

\section{Background}

Streptococcus agalactiae or group B Streptococcus (GBS) causes 147,000 stillbirths and infant deaths annually worldwide via maternal gastrointestinal and genital tract colonization [1, 2]. Maternal GBS colonization can result in early-onset disease (EOD), which may contribute to preterm delivery, maternal fever, and premature rupture of membrane (PROM) [3].

In 2010, the Centers for Disease Control and Prevention (CDC) advocated a universal culture-based screening for the early detection of GBS colonization during the 35th -37th gestational weeks, as well as the selective administration of intrapartum antibiotic prophylaxis (IAP) to prevent the incidence of EOD [4]. GBS culture remains the gold standard for the detection of GBS colonization. However, the typical turnaround time is $>24 \mathrm{~h}$, which makes it unsuitable for intrapartum screening. Moreover, it has relatively low sensitivity that ranges from $54-90 \%$ [5-8], and approximately $5-10 \%$ of non-hemolysis and/or nonpigmented GBS strains remain undetected [8-10]. In the past decade, nucleic acid amplification tests (NAATs), such as real-time fluorescence polymerase chain reaction (RT-PCR) [11] and the Cepheid Xpert 
real-time PCR assay [12], have been developed to accurately and sensitively detect GBS; however, their utility may be limited by the requirement of specialized instruments and well-trained personnel [11, 13]. The demand for instrument-free nucleic acid detection technologies has driven the development of isothermal amplification methods, such as real-time recombinase polymerase amplification (RT-RPA) [14] and real-time fluorescence loop-mediated isothermal amplification (RT-LAMP) assays [15]. Despite of easy-to-implement and time-saving, these methods are considerably less sensitive compared to RT-PCR based methods $[14,15]$. Therefore, a rapid and easy-to-implement method is required to facilitate the detection of GBS for intrapartum and antepartum screening.

Recently, CRISPR-Cas systems have provided the rapid, ultra-sensitive, and portable detection of nucleic acids [16-18]. These approaches rely on Cas proteins, which can be activated to cleave single-stranded DNA or RNA nonspecifically (also called cis-cleavage) after binding to a specific target via programmable CRISPR RNA (crRNA) [19-21]. In combination with nucleic acid pre-amplification techniques, such as PCR, recombinase polymerase amplification (RPA), and loop-mediated isothermal amplification (LAMP) [22-25], CRISPR-Cas systems have extremely high sensitivity of zM and exhibit specificities of 1-2 nt $[18,22]$. However, the application of the CRISPR-Cas system in the detection of urogenital tract bacteria, such as GBS, remains unexplored.

In this study, we developed a CRISPR-Cas12a-based assay for GBS detection, called the CRISPR-GBS assay. We conducted a retrospective, comparative study using vaginal or cervical swabs collected from 179 pregnant women with premature rupture of membrane (PROM), aiming to evaluate the clinical performance of the CRISPR-GBS assay by comparing with culture-based matrix-assisted laser desorption ionization time-of-flight (MALDI-TOF) mass spectrometry (culture-MS) method and RT-PCR assay.

\section{Results}

\section{Design and preparation of crRNAs for GBS detection}

We developed a rapid, high-sensitivity, and simple-to-use GBS detection assay by combining the RPA reaction with the CRISPR/Cas12a step, as shown in Fig. 1. The GBS genomic DNA (gDNA) was extracted from vaginal or cervical swabs by heating lysis. Then, a highly conserved target of the $c f b$ gene, which encodes the Christie-Atkins-Munch-Petersen (CAMP) factor, was selected to be amplified by RPA. Then, the crRNA-directed binding $c f b$ target activated the cis-cleavage activity of Cas $12 \mathrm{a}$, and the transcleavage of single-stranded DNA (ssDNA) produced a fluorescence signal. Within a short duration, the GBS-positive samples exhibited strong fluorescence signals compared to those in the negative samples.

To discriminate against other microorganisms, multiple sets of crRNAs adjacent to a 5'-TTTN-3' protospacer-adjacent motif (PAM) of the $c f b$ gene were designed, and their target efficiency were evaluated by a prediction score using the Cpf1-CRISPR-DT online software. Ultimately, 12 crRNAs with target efficiency scores $>0.3$ were included (Additional file 1 ). 
The more efficient crRNA adopted a conserved stem-loop structure [26] and showed lower minimum free energy (MFE) [27]. Therefore, we predicted the second structure and MFE of the 12 crRNAs using the NUPACK online tools (Additional file 2). Thus, five crRNAs (crRNA2, crRNA5, crRNA7, crRNA9, and crRNA12) were selected, and their efficiency were evaluated using GBS plasmid DNA concentrations of 5 copies/ $\mu \mathrm{L}$ (Fig. 2A) and 100 copies/ $\mu \mathrm{L}$ (Fig. 2B). Consequently, variable performance efficiency for each crRNA was observed, with crRNA9 showing the highest fluorescence signal at both 10 min and 45 min. Therefore, crRNA9 was selected for the remaining evaluations.

\section{Establishing the CRISPR-GBS assay}

RPA, an enrichment step of the detection target, can provide sufficient substates for crRNA binding and subsequent activation of the Cas12a protein. Thus, prior to the CRISPR assay establishment, several sets of conserved RPA primers targeting the $c f b$ gene of GBS were designed (Additional file 3). A set of primers, F3 and R1, showed the highest amplification efficiency among all the primer sets (Fig. 3A) and was adopted as the best primer pair. To further optimize our assay, we also investigated the appropriate concentration ratio of LbCas12a to crRNA. The results demonstrated that the 2: 1 ratio performed better than the others (Fig. 3B). Moreover, according to the manufacturer's instructions and considering our whole CRISPR-GBS assay workflow suitable for field-deployable diagnostics, we adopted a reaction temperature of $37^{\circ} \mathrm{C}$.

\section{Sensitivity of the CRISPR-GBS assay}

For the sensitivity of the CRISPR-GBS fluorescence assay, the limit of detection (LOD) was evaluated using the following concentrations: $10^{5}, 10^{4}, 10^{3}, 10^{2}, 10^{1}, 5$, and 1 copies/ $\mu \mathrm{L}$ of the diluted GBS plasmids in three independent trials. An equal value of molecular grade water served as the no-template control (NTC). Consequently, for DNA concentrations $>10^{2} \mathrm{copies} / \mu \mathrm{L}$, the plateau of the fluorescence signal reached within $10 \mathrm{~min}$, suggesting that the assay can be performed faster if required (Fig. 4). The LOD for CRISPR-GBS detection was 5 copies/ $\mu \mathrm{L}$ (Fig. 4). The fluorescence detection results showed that samples with concentrations of 5 copies/ $\mu \mathrm{L}$ or higher for the target plasmid DNA showed an obvious fluorescence signal compared to those of samples with lower concentrations and the negative control.

\section{Specificity of the CRISPR-GBS assay}

To assess the specificity of the CRISPR-GBS detection, gDNA extracted from GBS and other 16 microorganisms naturally found in the vaginal/anal flora were tested. Consequently, no positive results were obtained from the tested gDNA, except for the gDNA of the GBS strains (Fig. 5).

\section{Performance of CRISPR-GBS detection on the clinical samples}

A total of 179 vaginal or cervical swab specimens from pregnant women with PROM were collected to assess the clinical performance of the CRISPR-GBS assay using culture-MS as the reference method. The cut-off value of the CRISPR-GBS assay was determined by the Receiver operating characteristic (ROC) 
curve plotting (Additional file 4), which was obtained to be 53,259 (a.u.); that is, fluorescence signal values higher than 53,259 (a.u.) were considered positive, while the lower values were considered negative. Meanwhile, RT-PCR assay was established as a molecular comparative method. The LOD of our RT-PCR method was one copy per microliter, corresponding to the cycle threshold $\left(C_{T}\right)$ value of 25.8 (Fig. $6 A)$.

Of the 179 specimens, the CRISPR-GBS assay identified 144 GBS-positive samples out of 149 culture-MS positive specimens and correctly detected 30 culture-MS negative specimens (Table 1, Fig. 6B) Of the five undetected samples in our assay, four samples (nos. 52,62,63, and 85) were also undetectable by RTPCR, but one sample (no. 42) was GBS-positive by RT-PCR, with a $C_{T}$ value of 23.1, indicating a lower concentration of GBS gDNA in this sample (Table 2). Overall, our result showed a clinical sensitivity of $96.64 \%(144 / 149,95 \%$ confidence interval $[\mathrm{Cl}]=92.39-98.56 \%)$ and a clinical specificity of $100 \%$ $(30 / 30,95 \% \mathrm{Cl}=88.65-100 \%)$, and the high kappa value $(\mathrm{K})$ of $0.9061(P<0.001$; Table 1$)$ indicated a good correlation between the CRISPR-GBS assay and the culture-MS method (Table 1).

In the comparison of the CRISPR-GBS and RT-PCR assays, there were two discordant samples for GBS detection. In contrast to sample no. 42, sample no. 75 was identified as GBS-positive by the CRISPR-GBS but was undetected by RT-PCR assay (Table 2, Fig. 6B). Overall, the results demonstrated that CRISPRGBS was highly concordant with the RT-PCR assay, with an overall agreement percentage (OPA) of 98.88 $\%$, positive percentage agreement (PPA) of $99.31 \%$, and negative percentage agreement (NPA) of $97.14 \%$ (Table 3). Moreover, we also evaluated the difference in detection time between CRISPR-GBS and RT-PCR assay by calculating the mean with the corresponding SD of the time threshold for each of the 143 GBSpositive clinical lysates. The result showed that the mean detection time threshold for CRISPR-GBS assay was 2.9 min with an SD of $1.6 \mathrm{~min}$, resulting in the total amplification-to-detection time to be $17.9 \pm 1.6$ min (data not shown), whereas the total amplification-to-detection time threshold for RT-PCR was 39 min with an SD of $6.2 \mathrm{~min}$. Under similar sample preparation conditions, the CRISPR-GBS assay was $11.1 \mathrm{~min}$ faster than RT-PCR with all the steps performed at a constant temperature.

\section{Discussion}

To prevent neonatal disease, the rapid and reliable detection of GBS is essential to the timely administration of intrapartum antimicrobial chemoprophylaxis in pregnant women. According to the ASSURED criteria proposed by the World Health Organization (WHO) [28], the methods that are used to detect GBS should meet the requirements of point-of-care testing, particularly during delivery. In this study, we developed a CRISPR-based method for GBS detection by integrating RPA and Cas12a/crRNA trans-cleavage, namely, the CRISPR-GBS assay. We evaluated the analytical and clinical performance of the CRISPR-GBS assay. The CRISPR-GBS assay could detect GBS accurately without cross-reactivity with other non-target microorganisms. The LOD of the CRISPR-GBS assay was 5 copies $/ \mu \mathrm{L}$, which was consistent with one to three genomic copies for the RT-PCR assay [29], versus 98 genome copies per reaction for the RT-RPA assay [14]. Clinical evaluation using 179 specimens showed good performance compared with the culture-MS method and RT-PCR assay. 
The CRISPR-GBS assay is the first CRISPR-based system developed for GBS identification. The CRISPRGBS assay mainly distinguishes itself from the current systems, such as culture method, that is timeconsuming, or RT-PCR assay that requires standard PCR lab facilities and sophisticated instruments [29, 30]. The turnaround time of the CRISPR-GBS assay was within $35 \mathrm{~min}$, including $10 \mathrm{~min}$ for the DNA extraction step, $15 \mathrm{~min}$ for the RPA, and $10 \mathrm{~min}$ for the Cas12a/crRNA analysis. In case of the same DNA extraction procedure, the CRISPR-GBS assay only required an additional $25 \mathrm{~min}$, which was less than half of the running time of the RT-PCR assay ( $85 \mathrm{~min}$ ). In addition, we optimized the protocol by adding the CRISPR-Cas mixture to the lid of the RPA reaction tube, and then mixing it with the RPA products after transient centrifugation. The removal of the pipetting step not only shortens the time but also effectively avoids aerosol contamination. As for experimental conditions, the CRISPR-GBS assay requires only a constant temperature incubator and can be detected with lateral flow readouts if a real-time thermocycler is absent [31]. Moreover, the labor costs (equipment and personnel were not included) for the CRISPRGBS assay were estimated to be only $\$ 0.613$, which is lower than $\$ 4.95$ per swab of culture screening [32]. Thus, the CRISPR-GBS assay is suitable for large-scale antepartum screening and mass testing. A systematic comparison between the current GBS diagnostic assays is provided in Table 4.

The clinical performance of the 179 samples highlights the potential of the CRISPR-GBS assay as a promising assay for the identification of GBS, with a clinical sensitivity of $96.64 \%(144 / 149,95 \% \mathrm{Cl}=$ $92.39-98.56 \%)$ and a clinical specificity of $100 \%(30 / 30,95 \% \mathrm{Cl}=88.65-100 \%)$, compared with the culture-MS method. Culture-MS method as a reference has been confirmed to be more sensitive and specific than the conventional culture for GBS identification [8] [33-35]. Moreover, CRISPR-GBS demonstrated a high concordance of $98.88 \%$ with the RT-PCR assay. There was a total of six discordant samples between culture-MS and the two molecular assays - CRISPR-GBS and RT-PCR (Table 2). These discrepancies might be attributed to the use of different swabs with variable bacterial loads that were obtained from per participant, especially for individuals carrying small amounts of bacteria. Moreover, the presence of inhibitors that block amplification or DNA degradation before RPA may result in a falsenegative result in the molecular methods [36,37]. Of note, all of these six discordant samples were collected from the cervix of women with PROM, which could lead to a substantial reduction of the coccus per swab compared with the swabbing from both the lower vagina and rectum [4].

A limitation of this study is that it is difficult to quantify the true GBS bacterial load in clinical samples. The DNA extraction is subjected to amplification using RPA prior to CRISPR-Cas12a processing, which rapidly reaches a signal plateau owing to its high efficiency. Additionally, the lack of internal amplification control in our assay does not allow to determine whether RPA is blocked by some inhibitors. Thus, a CRISPR-based duplex detection system containing the internal amplification control to verify the efficiency should be explored in future work.

\section{Conclusions}

There is an urgent need for the timely detection of GBS infection for pregnant women with PROM. This study developed a CRISPR-GBS assay as a promising detection method that is rapid, easy to use, cost 
effective, and robust. The assay allows clinicians to determine the most suitable options for intrapartum antibiotic prophylaxis during delivery.

\section{Methods}

\section{Oligonucleotide design and synthesis}

The DNA sequence of $c f b$ (GenBank accession No. X72754.1) was retrieved from the NCBI website (http://www.ncbi.nlm.nih.gov) and cloned into the pUC57 vector to generate the GBS plasmid pUC57$c f b$. The RPA primers were designed specifically for the $c f b$ gene, and the RPA assay was performed using a TwistAmp ${ }^{T M}$ Basic kit (TwistDx, Cambridge, UK), according to the manufacturer's protocol. The ssDNA reporter labelling with FAM and BHQ-3 was used for the Cas12a/crRNA reaction. For the RT-PCR assay, the forward and reverse primers as well as the probe, which target the $c f b$ gene as previously described [14] was used in this study. All primers, DNA/RNA oligonucleotides, and probes were synthesized by Sangon Biotech (Shanghai, China), and the sequence information is listed in detail in Additional file 3.

\section{Design and preparation of the crRNAs}

The crRNAs were designed according to the target sequence of the $c f b$ gene, and their target efficiency were scored using the CRISPR-DT online software (http://bioinfolab.miamioh.edu/CRISPRDT/interface/Cpf1_efficiency.php) for Cpf1. The secondary structure and the MFE of the crRNAs were further evaluated using NUPACK (http://www.nupack.org/). Then, the homology of these crRNAs was analyzed using Nucleotide BLAST (https://blast.ncbi.nlm.nih.gov/Blast.cgi). The selected crRNAs were synthesized by Sangon Biotech (Shanghai, China). The crRNA that exhibited the highest efficiency was used in the subsequent detection assay.

\section{CRISPR/Cas12a fluorescence assay}

RPA was performed using a TwistAmp ${ }^{T M}$ Basic kit (TwistDx, Cambridge, UK). Briefly, $12.5 \mu \mathrm{L}$ of the total reaction volume was made up of $1 \times$ rehydration buffer, $0.48 \mathrm{mmol} / \mathrm{L}$ of the forward and reverse primers, $2.5 \mu \mathrm{L}$ of the gDNA, $1 \mu \mathrm{L}$ of MgOAc $(280 \mathrm{mM})$, RPA mix, and diethylpyrocarbonate water up to $12.5 \mu \mathrm{L}$. The reaction was incubated at $37^{\circ} \mathrm{C}$ for $15 \mathrm{~min}$, including a manual mixing step (5-s tube vortex) for 4 min. For the NTC, these reactions were prepared by substituting the target DNA with an equal volume of molecular grade water.

After RPA reaction, $12.5 \mu \mathrm{L}$ of RPA products mixed with $7.5 \mu \mathrm{L}$ of Cas12a reaction mixture, which contains $2 \mu \mathrm{L}$ of crRNA $(1 \mu \mathrm{M}), 1 \mu \mathrm{L}$ of DNA reporter $(10 \mu \mathrm{M}), 1 \mu \mathrm{L}$ of LbaCas $12 \mathrm{a}(1 \mu \mathrm{M}), 2 \mu \mathrm{L}$ of $1 \times$ NEBuffer 2.0 (New England Biolabs, UK), and $1.5 \mu \mathrm{L}$ of RNase-free water, formed the final volume of 20 $\mu \mathrm{L}$. We used commercial LbaCas12a (EnGen LbaCas12a, M0653T, NEB, NE). Then, the reaction was incubated at $37^{\circ} \mathrm{C}$ for 10 min using the Applied Biosystems ${ }^{T M}$ QuantStudio 3 (ThermoFisher Scientific, MA, USA) and the fluorescence values were measured every minute. 


\section{Analytical studies}

For the specificity assay, Streptococcus agalactiae (ATCC 12386) and other 16 microorganisms (Fig. 5) that are naturally found in the vagina were obtained from the Department of Microbiology Laboratory, Fujian Maternity and Child Health Hospital (Fuzhou, China). All strains were suspended in $1 \mathrm{~mL}$ of TE buffer (10 mM Tris-HCl, pH8.5, 1 mM EDTA, and 1\% TritonX-100). The gDNA was extracted by heating lysis at $100{ }^{\circ} \mathrm{C}$ for $10 \mathrm{~min}$, and the supernatants were collected by centrifugation at $12,000 \times \mathrm{g}$ for $10 \mathrm{~min}$. The concentration of the gDNA was quantified using a NanoDrop \& Qubit 4 spectrophotometer (Thermo Fisher, US) and diluted to $1 \times 10^{5}$ genome copies for use. Then, $2.5 \mu \mathrm{L}$ of each DNA template was added to the reaction mixtures for RPA.

For the sensitivity assay of the CRISPR-GBS fluorescence detection, the GBS plasmid pUC57-cfb was quantified using a NanoDrop \& Qubit 4 spectrophotometer (Thermo Fisher, US) and serially diluted in RNase-free water to $10^{5}, 10^{4}, 10^{3}, 10^{2}, 10^{1}, 5$, and 1 copies/ $\mu \mathrm{L}$, respectively. Then, $2.5 \mu \mathrm{L}$ of various concentrations of the GBS plasmid DNA template were added to the RPA mixture for amplification. Then, $12.5 \mu \mathrm{L}$ of the RPA product was transferred into the Cas12a reaction mixture. For the LOD analysis, the number of replicates for each dilution was at least 12 at three different times. The LOD was determined by the lowest concentration that gave no more than one negative result.

\section{Culture-MS method identification of GBS}

Vaginal or cervical swabs were inoculated onto column blood agar (Bioivd, China) at $37^{\circ} \mathrm{C}$ in $5 \% \mathrm{CO}_{2}$ for 18-24 h. Negative plates were incubated for an additional $24 \mathrm{~h}$ prior to signing out. Then, the presumptive, beta-hemolytic GBS colonies were selected to undergo a confirmatory test by MALDI-TOF mass spectrometry (Bruker Biotyper, BD). The culture-MS identification was completed by the medical workers in the bacteriological laboratory of Fujian Maternity and Child Health Hospital.

\section{Clinical study of CRISPR-GBS Assay}

A total of 179 vaginal or cervical swab specimens from pregnant women with PROM were collected from 2020 to 2021 at the Fujian Maternity and Child Health Hospital (Fuzhou, China). All these 179 specimens were obtained in duplicate from each participant, one for culture-MS assay performed in the microbiology laboratory, and another one was dedicated to DNA extraction by heating lysis, as described previously (see Methods). The supernatant was used as the gDNA for the CRISPR-GBS and RT-PCR assay, which was performed on the Applied Biosystems ${ }^{T M}$ QuantStudio 3 (ThermoFisher Scientific, MA, USA). The clinical performance of the CRISPR-GBS assay was evaluated by comparing the culture-MS and RTPCR results.

\section{Determination of cut-off value for the CRISPR-GBS assay}

For analysis of the clinical performance of the CRISPR-GBS assay, the ROC and Youden index were used to determine the optimal cut-off value. Fluorescence signal value higher than the cut-off value was 
considered positive, while the lower value was considered negative. We randomly selected a total of 30 clinical samples, containing 15 negative samples and 15 positive samples that were tested by the cultureMS method. The cut-off value was determined by the maximum Youden index, which was calculated using the following formula: Youden index = sensitivity + specificity - 1. ROC was statistically analyzed using the GraphPad Prism software (version 5.0), while the Youden Index was determined using Microsoft Excel (2016).

\section{Conventional RT-PCR assays for GBS detection}

RT-PCR was used to validate the CRISPR-GBS assay. It was performed in a total volume of $25 \mu \mathrm{L}$, containing $0.2 \mu \mathrm{L}$ of Taq (Hot Start Version; Takara Bio, Japan), $25 \mathrm{mM}$ of $\mathrm{MgCl}_{2}, 2.5 \mathrm{mM}$ of dNTP, 0.2 $\mu \mathrm{M}$ of TaqMan probe, $0.16 \mu \mathrm{M}$ of the forward primer, $0.16 \mu \mathrm{M}$ of the reverse primer, $11 \mu \mathrm{L}$ of PCR-grade water, $1 \times$ PCR buffer (Takara Bio, Japan), and $5 \mu \mathrm{L}$ of the DNA. A touchdown RT-PCR method was performed as follows: after an initial denaturation step at $95^{\circ} \mathrm{C}$ for $10 \mathrm{~min}, 10$ cycles at $95^{\circ} \mathrm{C}$ for $30 \mathrm{~s}$ and $65^{\circ} \mathrm{C}$ for $60 \mathrm{~s}\left(-1^{\circ} \mathrm{C}\right.$ per cycle), followed by 40 cycles at $95^{\circ} \mathrm{C}$ for $30 \mathrm{~s}$ and $55^{\circ} \mathrm{C}$ for $60 \mathrm{~s}$ was performed using the Applied Biosystems ${ }^{T M}$ QuantStudio 3 (ThermoFisher Scientific, MA, USA). The NTC reactions substituted with equal values of molecular grade water were conducted simultaneously. The results were considered positive when the $C T$ value was $>0$ and $\leq 25.83$.

\section{Data analysis}

All the results that were generated from at least three independent experiments are presented as the mean \pm standard deviation (SD) and compared using Student's $t$ test. Statistical significance was set at $P<$ 0.05. Statistical analyses and graphing were conducted using Origin Lab version 8.0, GraphPad prism 5.0, and Adobe Illustrator CS5. The sensitivity, specificity, K, PPV, and NPV agreement with two-sided $95 \% \mathrm{Cls}$ were analyzed using OpenEpi (http://wwww.openepi.com/Menu/OE_Menu.htm).

\section{List Of Abbreviations}

CAMP, Christie-Atkins-Munch-Petersen; Cas, CRISPR-associated protein; CDC, Centers for Disease Control and Prevention; $\mathrm{Cl}$, confidence interval; $\mathrm{CRISPR}$, clustered regularly interspaced short palindromic repeats; CT, cycle threshold; GBS, group B Streptococcus; gDNA, genomic DNA; IAP, intrapartum antibiotic prophylaxis; LAMP, loop-mediated isothermal amplification; LOD, limit of detection; MALDI-TOF, matrixassisted laser desorption ionization time-of-flight; MFE, minimum free energy; NAAT, nucleic acid amplification test; NPA, negative percentage agreement; NTC, no-template control; OPA, overall agreement percentage; PAM, protospacer-adjacent motif; PROM, premature rupture of membrane; PPA, positive percentage agreement; ROC, receiver operating characteristic; RPA, recombinase polymerase amplification; RT-PCR, real-time fluorescence polymerase chain reaction; RT-RPA, real-time recombinase polymerase amplification; RT-LAMP, real-time fluorescence loop-mediated isothermal amplification; SD, standard deviation; ssDNA, single-stranded DNA; WHO, World Health Organization. 


\section{Declarations}

\section{Ethics approval and consent to participate}

This study was approved by the Ethics Committee of the Fujian Maternity and Child Care Hospital (ID: No. 2021KR09032). All methods were carried out in accordance with Declaration of Helsinki. Informed consent was obtained from all the participants prior to enrollment.

\section{Consent for publication}

Not applicable.

\section{Availability of data and materials}

The raw data during the current study are available from figshare

(https://doi.org/10.6084/m9.figshare.17149208.v1)

\section{Competing interests}

The authors declare that they have no competing interests.

\section{Funding}

This study was supported by grants from the Fujian Maternity and Child Health Hospital Research Fund Project (YCXM 20-30), Fujian Provincial Natural Science Foundation (2020J05275)

\section{Authors' contributions}

DY and XW conceived and designed the study. HX participated in primer design. ZW contributed to specimen collection. HX and ZY analyzed the data. DY and BL wrote the initial draft of the manuscript. $\mathrm{BL}$ and $\mathrm{XW}$ revised the manuscript. All authors read and approved the final manuscript.

\section{Acknowledgements}

We thank all the medical workers in both the Medical Research Center, Fujian Maternity and Child Health Hospital and the Clinical laboratory, Fujian Maternity and Child Health Hospital, for their cooperation in this study.

\section{Author details}

${ }^{1}$ Fujian Obstetrics and Gynecology Hospital, Fuzhou, Fujian 350011, China. ${ }^{2}$ Medical Research Center, Fujian Maternity and Child Health Hospital, Affiliated Hospital of Fujian Medical University, Fuzhou, Fujian 350001, China. ${ }^{3}$ Key Laboratory of Technical Evaluation of Fertility Regulation of Non-human Primate, National Health Commission, Fujian Maternity and Child Health Hospital, Affiliated Hospital of Fujian Medical University, Fuzhou, Fujian 350001, China. ${ }^{4}$ Medical Genetic Diagnosis and Therapy Center, Fujian 
Key Laboratory for Prenatal Diagnosis and Birth Defect, Fujian Maternity and Child Health Hospital, Affiliated Hospital of Fujian Medical University, Fuzhou, Fujian 350001, China. ${ }^{5}$ The United Innovation of Mengchao Hepatobiliary Technology Key Laboratory of Fujian Province, Mengchao Hepatobiliary Hospital of Fujian Medical University, Fuzhou, Fujian 350025, China. ${ }^{6}$ College of Chemical Engineering, Fuzhou University, Fuzhou, Fujian 350116, China. ${ }^{7}$ Department of Clinical Laboratory, Fujian Maternity and Child Health Hospital, Affiliated Hospital of Fujian Medical University, Fuzhou, Fujian 350001, China.

\section{References}

1. Seale AC, Bianchi-Jassir F, Russell NJ, Kohli-Lynch M, Tann CJ, Hall J, Madrid L, Blencowe H, Cousens S, Baker CJ et al. Estimates of the Burden of Group B Streptococcal Disease Worldwide for Pregnant Women, Stillbirths, and Children. Clin Infect Dis. 2017; 65(suppl_2):S200-S219.

2. Russell NJ, Seale AC, O'Driscoll M, O'Sullivan C, Bianchi-Jassir F, Gonzalez-Guarin J, Lawn JE, Baker CJ, Bartlett L, Cutland C et al: Maternal Colonization With Group B Streptococcus and Serotype Distribution Worldwide: Systematic Review and Meta-analyses. Clin Infect Dis. 2017; 65(suppl_2):S100-S111.

3. Edmond KM, Kortsalioudaki C, Scott S, Schrag SJ, Zaidi AKM, Cousens S, Heath PT. Group B streptococcal disease in infants aged younger than 3 months: systematic review and meta-analysis. The Lancet. 2012; 379(9815):547-56.

4. Verani JR, McGee L, Schrag SJ; Division of Bacterial Diseases, National Center for Immunization and Respiratory Diseases, Centers for Disease Control and Prevention (CDC). Prevention of perinatal group B streptococcal disease-revised guidelines from CDC, 2010. MMWR Recomm Rep. 2010;59(RR-10):1-36.

5. Couturier BA, Weight T, Elmer H, Schlaberg R. Antepartum screening for group B Streptococcus by three FDA-cleared molecular tests and effect of shortened enrichment culture on molecular detection rates. J Clin Microbiol. 2014;52(9):3429-32.

6. Miller SA, Deak E, Humphries R. Comparison of the AmpliVue, BD Max System, and illumigene Molecular Assays for Detection of Group B Streptococcus in Antenatal Screening Specimens. J Clin Microbiol. 2015; 53(6):1938-41.

7. Salem N, Anderson JJ. Evaluation of four chromogenic media for the isolation of Group B Streptococcus from vaginal specimens in pregnant women. Pathology. 2015; 47(6):580-2.

8. Salimnia H, Robinson-Dunn B, Gundel A, Campbell A, Mitchell R, Taylor M, Fairfax MR. Suggested Modifications To Improve the Sensitivity and Specificity of the 2010 CDC-Recommended Routine Streptococcus agalactiae Screening Culture for Pregnant Women. J Clin Microbiol. 2019;57(8).

9. Schrag SJ. The past and future of perinatal group B streptococcal disease prevention. Clin Infect Dis. 2004;39(8):1136-8.

10. Nickmans S, Verhoye E, Boel A, Van Vaerenbergh K, De Beenhouwer H. Possible solution to the problem of nonhemolytic group B streptococcus on granada medium. J Clin Microbiol. 2012; 
50(3):1132-3.

11. Ke D, Ménard C, Picard FJ, et al. Development of conventional and real-time PCR assays for the rapid detection of group B streptococci. Clin Chem. 2000;46(3):324-31.

12. Bourgeois-Nicolaos N, Cordier AG, Guillet-Caruba C, Casanova F, Benachi A, Doucet-Populaire F. Evaluation of the Cepheid Xpert GBS assay for rapid detection of group B Streptococci in amniotic fluids from pregnant women with premature rupture of membranes. J Clin Microbiol. 2013; 51(4):1305-6.

13. Zeng YF, Chen CM, Li XY, Chen JJ, Wang YG, Ouyang S, Ji TX, Xia Y, Guo XG. Development of a droplet digital PCR method for detection of Streptococcus agalactiae. BMC Microbiol. 2020; 20(1):179.

14. Daher RK, Stewart G, Boissinot M, Bergeron MG. Isothermal recombinase polymerase amplification assay applied to the detection of group B streptococci in vaginal/anal samples. Clin Chem. 2014; 60(4):660-6.

15. Guo XG, Zhuang YR, Wen JZ, Xie TA, Liu YL, Zhu GD, Xia Y. Evaluation of the real-time fluorescence loop-mediated isothermal amplification assay for the detection of Streptococcus agalactiae. Biosci Rep. 2019; 39(5).

16. Abudayyeh OO, Gootenberg JS. CRISPR diagnostics. 2021; 372(6545):914-5.

17. Li Y, Li S, Wang J, Liu G. CRISPR/Cas Systems towards Next-Generation Biosensing. Trends Biotechnol. 2019;37(7):730-43.

18. Kellner MJ, Koob JG, Gootenberg JS, Abudayyeh OO, Zhang F. SHERLOCK: nucleic acid detection with CRISPR nucleases. Nat Protoc. 2019; 14(10):2986-3012.

19. Abudayyeh 00 , Gootenberg JS, Konermann $\mathrm{S}$, et al. C2c2 is a single-component programmable RNAguided RNA-targeting CRISPR effector. Science. 2016;353(6299):aaf5573.

20. Li SY, Cheng QX, Liu JK, Nie XQ, Zhao GP, Wang J. CRISPR-Cas12a has both cis- and trans-cleavage activities on single-stranded DNA. Cell Res. 2018;28(4):491-3.

21. Chen JS, Ma E, Harrington LB, et al. CRISPR-Cas12a target binding unleashes indiscriminate singlestranded DNase activity [published correction appears in Science. 2021 Feb 19;371(6531):]. Science. 2018;360(6387):436-9.

22. Gootenberg JS, Abudayyeh 00, Lee JW, Essletzbichler P, Dy AJ, Joung J, Verdine V, Donghia N, Daringer NM, Freije CA et al. Nucleic acid detection with CRISPR-Cas13a/C2c2. Science. 2017; 356(6336):438-42.

23. Gootenberg JS, Abudayyeh 00 , Kellner MJ, Joung J, Collins JJ, Zhang F. Multiplexed and portable nucleic acid detection platform with Cas13, Cas12a, and Csm6. Science. 2018; 360(6387):439-44.

24. Wang S, Li H, Kou Z, Ren F, Jin Y, Yang L, Dong X, Yang M, Zhao J, Liu H et al. Highly sensitive and specific detection of hepatitis $B$ virus DNA and drug resistance mutations utilizing the PCR-based CRISPR-Cas13a system. Clin Microbiol Infect. 2021; 27(3):443-50. 
25. Garcia-Venzor A, Rueda-Zarazua B, Marquez-Garcia E, Maldonado V, Moncada-Morales A, Olivera H, Lopez I, Zuniga J, Melendez-Zajgla J. SARS-CoV-2 Direct Detection Without RNA Isolation With LoopMediated Isothermal Amplification (LAMP) and CRISPR-Cas12. Front Med (Lausanne). 2021; 8:627679.

26. Yamano T, Nishimasu H, Zetsche B, Hirano H, Slaymaker IM, Li Y, Fedorova I, Nakane T, Makarova KS, Koonin EV et al. Crystal Structure of Cpf1 in Complex with Guide RNA and Target DNA. Cell. 2016;165(4):949-62.

27. Ke Y, Huang S, Ghalandari B, Li S, Warden AR, Dang J, Kang L, Zhang Y, Wang Y, Sun Y et al. HairpinSpacer crRNA-Enhanced CRISPR/Cas13a System Promotes the Specificity of Single Nucleotide Polymorphism (SNP) Identification. Adv Sci (Weinh) 2021, 8(6):2003611.

28. Peeling RW, Holmes KK, Mabey D, Ronald A. Rapid tests for sexually transmitted infections (STIs): the way forward. Sex Transm Infect. 2006; 82 Suppl 5:v1-6.

29. Ke D, Ménard C, Picard FJ, et al. Development of conventional and real-time PCR assays for the rapid detection of group B streptococci. Clin Chem. 2000;46(3):324-31.

30. Clarke C, O'Connor L, Carre-Skinner H, Piepenburg O, Smith TJ. Development and performance evaluation of a recombinase polymerase amplification assay for the rapid detection of group $B$ streptococcus. BMC Microbiol. 2016; 16(1):221.

31. Yu F, Zhang K, Wang Y, Li D, Cui Z, Huang J, Zhang S, Li X, Zhang L. CRISPR/Cas12a-based on-site diagnostics of Cryptosporidium parvum Ild-subtype-family from human and cattle fecal samples. Parasit Vectors. 2021; 14(1):208.

32. Otaguiri ES, Morguette AEB, Morey AT, Tavares ER, Kerbauy G, de Almeida Torres RSL, Chaves Junior $M$, Tognim MCB, Goes VM, Krieger MA et al. Development of a melting-curve based multiplex realtime PCR assay for simultaneous detection of Streptococcus agalactiae and genes encoding resistance to macrolides and lincosamides. BMC Pregnancy Childbirth. 2018; 18(1):126.

33. To KN, Cornwell E, Daniel R, Goonesekera S, Jauneikaite E, Chalker V, Le Doare K. Evaluation of matrix-assisted laser desorption ionisation time-of-flight mass spectrometry (MALDI-TOF MS) for the Identification of Group B Streptococcus. BMC Res Notes. 2019;12(1):85.

34. Alatoom AA, Cunningham SA, Ihde SM, Mandrekar J, Patel R. Comparison of direct colony method versus extraction method for identification of gram-positive cocci by use of Bruker Biotyper matrixassisted laser desorption ionization-time of flight mass spectrometry. J Clin Microbiol. 2011; 49(8):2868-73.

35. Lartigue MF, Kostrzewa M, Salloum M, Haguenoer E, Hery-Arnaud G, Domelier AS, Stumpf S, Quentin R. Rapid detection of "highly virulent" Group B Streptococcus ST-17 and emerging ST-1 clones by MALDI-TOF mass spectrometry. J Microbiol Methods. 2011; 86(2):262-5.

36. Al-Soud WA, Radstrom P. Purification and characterization of PCR-inhibitory components in blood cells. J Clin Microbiol. 2001; 39(2):485-93.

37. de Zoysa A, Edwards K, Gharbia S, Underwood A, Charlett A, Efstratiou A: Non-culture detection of Streptococcus agalactiae (Lancefield group B Streptococcus) in clinical samples by real-time PCR. J 
Med Microbiol. 2012; 61(Pt 8):1086-90.

\section{Tables}

Table 1

Performance of CRISPR-GBS assay in clinical samples compared with culture-MS method Culture-MS method Comparison of two methods

\begin{tabular}{lllllll|}
$\begin{array}{l}\text { CRISPR-GBS } \\
\text { assay }\end{array}$ & Positive & Negative & Total & \%Sensitivity & \%Specificity & Kappa \\
\hline Positive & 144 & 0 & 144 & $96.64(92.39-$ & $\begin{array}{l}100 \\
(88.65-\end{array}$ & $\begin{array}{l}0.9061 \\
(0.7603-\end{array}$ \\
& & & & $98.56)^{a}$ & $100)$ & $1.052)$
\end{tabular}

$\begin{array}{llll}\text { Negative } & 5 & 30 & 35 \\ \text { Total } & 149 & 30 & 179\end{array}$

a Values in parentheses are the $95 \% \mathrm{Cl}$, two-sided $95 \%$ confidence interval. Abbreviations. CRISPR-GBS, CRISPR-based method for GBS detection; Culture-MS, culture method combined with matrix-assisted laser desorption/ionization time-off light mass spectrometry

Table 2

Details of the six discordant samples among CRISPR-GBS, RT-PCR, and culture-MS method

\begin{tabular}{|lllll|}
\hline \multicolumn{5}{|c|}{ Individual test result } \\
\hline Sample no. & Source & CRISPR-GBS & RT-PCR $^{\text {b }}$ (C T value) & Culture-MS result \\
\hline 42 & cervix & Negative & Positive $(23.11)$ & Positive \\
\hline 52 & cervix & Negative & Undetected (0) & Positive \\
\hline 62 & cervix & Negative & Undetected (0) & Positive \\
\hline 63 & cervix & Negative & Negative $(32.64)$ & Positive \\
\hline 75 & cervix & $784806.94^{a}$ & Negative $(31.79)$ & Positive \\
\hline 85 & cervix & Negative & Negative $(26.59)$ & Positive \\
\hline${ }^{a}$ Fluorescence signal value (a.u). & & \\
\hline${ }^{b}$ RT-PCR result was judged as positive when the cycle threshold $\left(C_{T}\right)$ value was $>0$ and $\leq 25.83$. \\
\hline
\end{tabular}


Table 3

Comparison of the clinical performance of the CRISPR-GBS with RT-PCR assay

CRISPR-GBS

\begin{tabular}{|c|c|c|c|c|c|c|}
\hline RT-PCR & Positive & Negative & Total & $\% \mathrm{OPA}^{a}$ & $\% \mathrm{PPA}^{\mathrm{b}}$ & $\% N^{\prime} A^{c}$ \\
\hline Positive & 143 & 1 & 144 & $\begin{array}{l}98.88(96.02- \\
99.69)^{d}\end{array}$ & $\begin{array}{l}99.31(96.17- \\
99.88)\end{array}$ & $\begin{array}{l}97.14(85.47- \\
99.49)\end{array}$ \\
\hline Negative & 1 & 34 & 35 & & & \\
\hline Total & 144 & 35 & 179 & & & \\
\hline
\end{tabular}

${ }^{a}$ OPA, Overall percent agreement.

${ }^{b}$ PPA, Positive percent agreement.

${ }^{c}$ NPA, Negative percent agreement.

${ }^{d}$ Values in parentheses are the $95 \% \mathrm{Cl}$, two-sided $95 \%$ confidence interval.

Comparison of two methods

Table 4

Comparison of the CRISPR-GBS assay with several current GBS detection methods

\begin{tabular}{|c|c|c|c|c|}
\hline & RT-PCR [29] & RT-LAMP [15] & RT-RPA[14] & CRISPR-GBS \\
\hline LOD & $\begin{array}{l}1-3 \text { genome } \\
\text { copies }\end{array}$ & $300 \mathrm{pg} / \mu \mathrm{L}$ & $\begin{array}{l}98 \text { genomic } \\
\text { copies }\end{array}$ & 5 copies/ $\mu \mathrm{L}$ \\
\hline Cross-react & No & No & No & No \\
\hline $\begin{array}{l}\text { Turnaround } \\
\text { time }\end{array}$ & $\begin{array}{l}\text { Detection time of } \\
35 \text { min (exclude } \\
\text { DNA extraction) }\end{array}$ & $\begin{array}{l}\text { Detection time of } \\
60 \text { min (exclude } \\
\text { DNA extraction) }\end{array}$ & $\begin{array}{l}\text { Detection time of } \\
30 \text { min (exclude } \\
\text { DNA extraction) }\end{array}$ & $\begin{array}{l}\text { Turnaround time of } \\
35 \text { min (Include } \\
\text { DNA extraction) }\end{array}$ \\
\hline $\begin{array}{l}\text { DNA } \\
\text { extraction }\end{array}$ & $\begin{array}{l}\text { DNA extraction kit } \\
\text { (G NOME kit) }\end{array}$ & DNA extraction kit & $\begin{array}{l}\text { DNA extraction kit } \\
\text { (BD GeneOhmTM } \\
\text { Strep B kit) }\end{array}$ & Heating lysis \\
\hline Target & $c f b$ gene & fbs gene & $c f b$ gene & $c f b$ gene \\
\hline Temperature & $\begin{array}{l}\text { Temperature } \\
\text { cycles }\end{array}$ & $\begin{array}{l}\text { A constant } \\
\text { temperature of } 63 \\
{ }^{\circ} \mathrm{C}\end{array}$ & $\begin{array}{l}\text { A constant } \\
\text { temperature of } 39 \\
{ }^{\circ} \mathrm{C}\end{array}$ & $\begin{array}{l}\text { A constant } \\
\text { temperature of } 37 \\
{ }^{\circ} \mathrm{C}\end{array}$ \\
\hline $\begin{array}{l}\text { Clinical } \\
\text { samples }\end{array}$ & 15 vaginal swabs & No & $\begin{array}{l}50 \text { vaginal/anal } \\
\text { samples }\end{array}$ & $\begin{array}{l}179 \text { vaginal/ } \\
\text { cervical swabs }\end{array}$ \\
\hline Cost & Unknown & Unknown & Around \$ 10 & Around \$ 0.6138 \\
\hline
\end{tabular}

Figures 


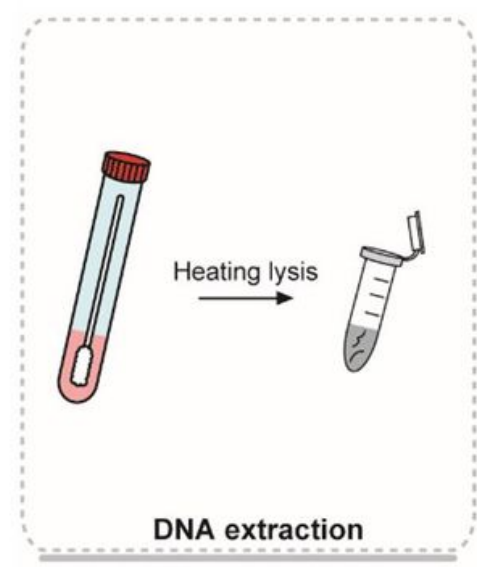

$100^{\circ} \mathrm{C} 10 \mathrm{~min}$

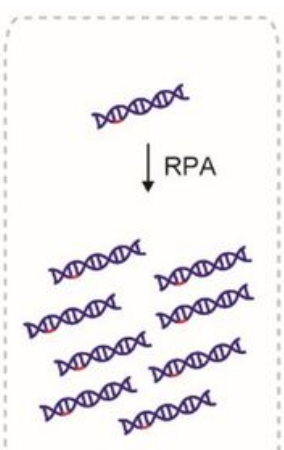

Amplification

$37^{\circ} \mathrm{C} 15 \mathrm{~min}$

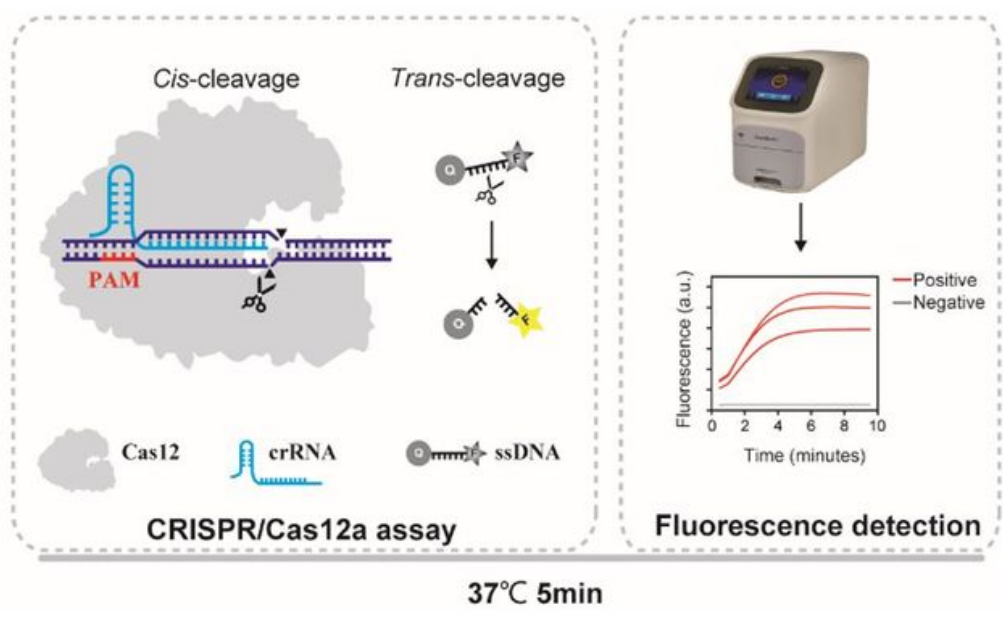

$37^{\circ} \mathrm{C} 5 \mathrm{~min}$

\section{Figure 1}

Schematic diagram of the CRISPR-GBS assay. Genomic DNA was extracted and the target gene was amplified by recombinase polymerase amplification (RPA). Positive fluorescence signals were produced when ssDNA probes were trans-cleaved by activated Cas12a when the crRNA recognized the PAM of the target sequence. PAM, protospacer-adjacent motif; ssDNA, single-stranded DNA.

A

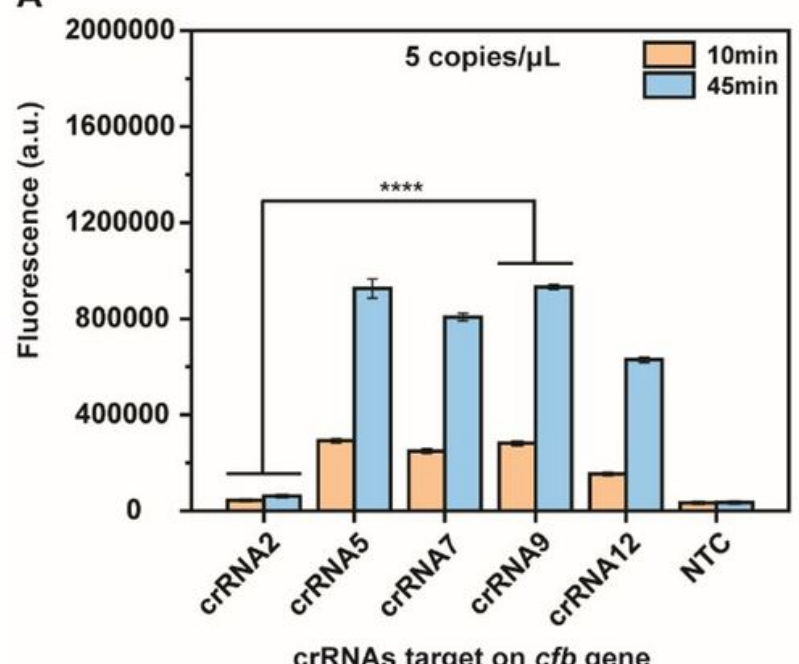

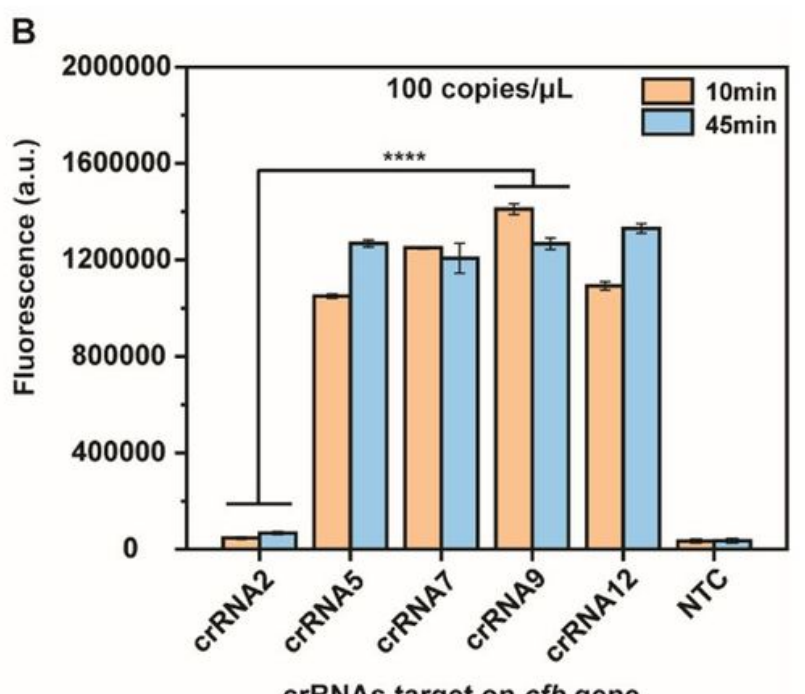

crRNAs target on cfb gene

\section{Figure 2}

Screening for highly active crRNA. The efficiency of five crRNAs targeting the cfb gene were evaluated at GBS plasmid DNA concentrations of 5 copies/ $\mu \mathrm{L}(\mathrm{A})$ and 100 copies/ $\mu \mathrm{L}$ (B) for 10 min (orange color) and $45 \mathrm{~min}$ (blue color). Fluorescence signal reflects the activation of the individual crRNA, $n=3$ technical replicates; error bars represent mean \pm SD; $* * * * p \leq 0.0001$. 

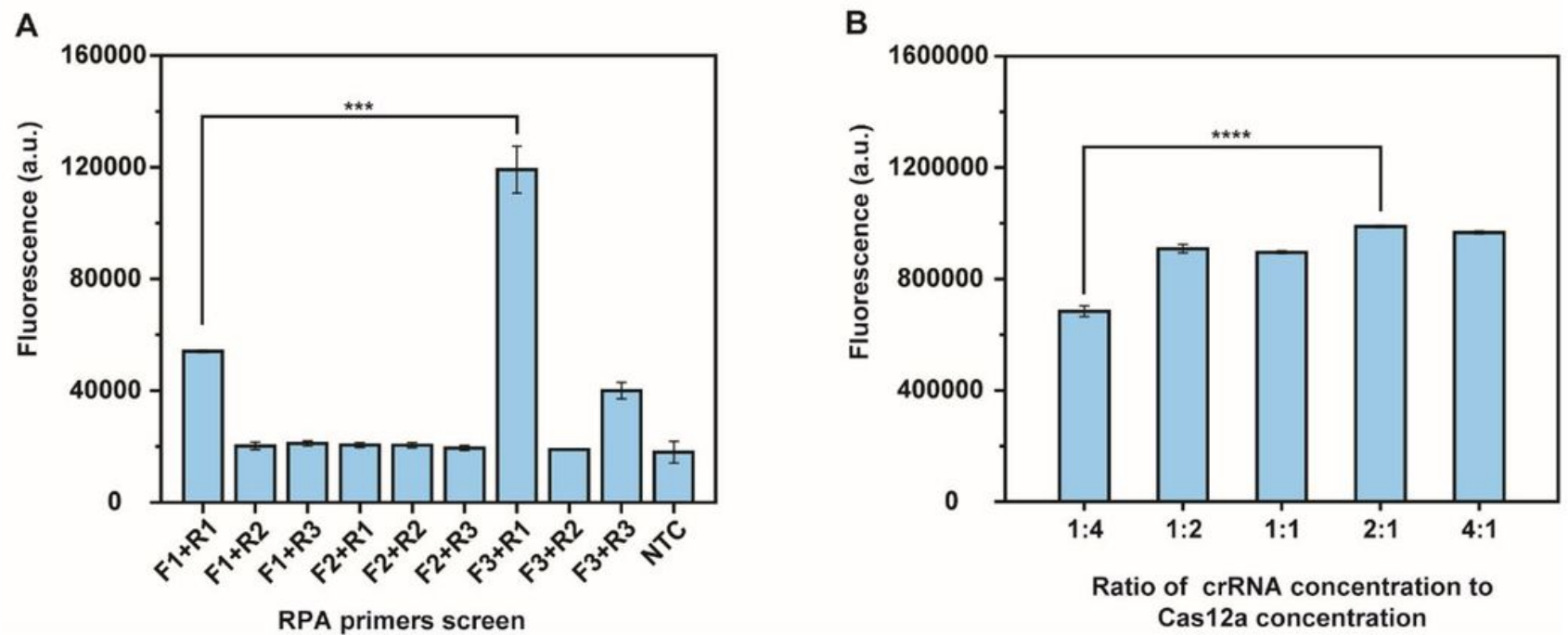

Figure 3

Optimization of CRISPR-GBS fluorescence assay. (A) Determination of optimal primer sets. We designed nine primer sets to amplify the cfb gene with 100 copies/ $\mu$ L DNA template for CRISPR-GBS fluorescence assay at 10 min. (B) Optimizing the concentration ratio of crRNA to LbCas12a for CRISPR-GBS fluorescence assay at $10 \mathrm{~min}$. Error bars in panels $(A, B)$ represent the mean $\pm S D, n=3$ replicates. $* * * p$ $\leq 0.001$ and $* * * * p \leq 0.0001$.

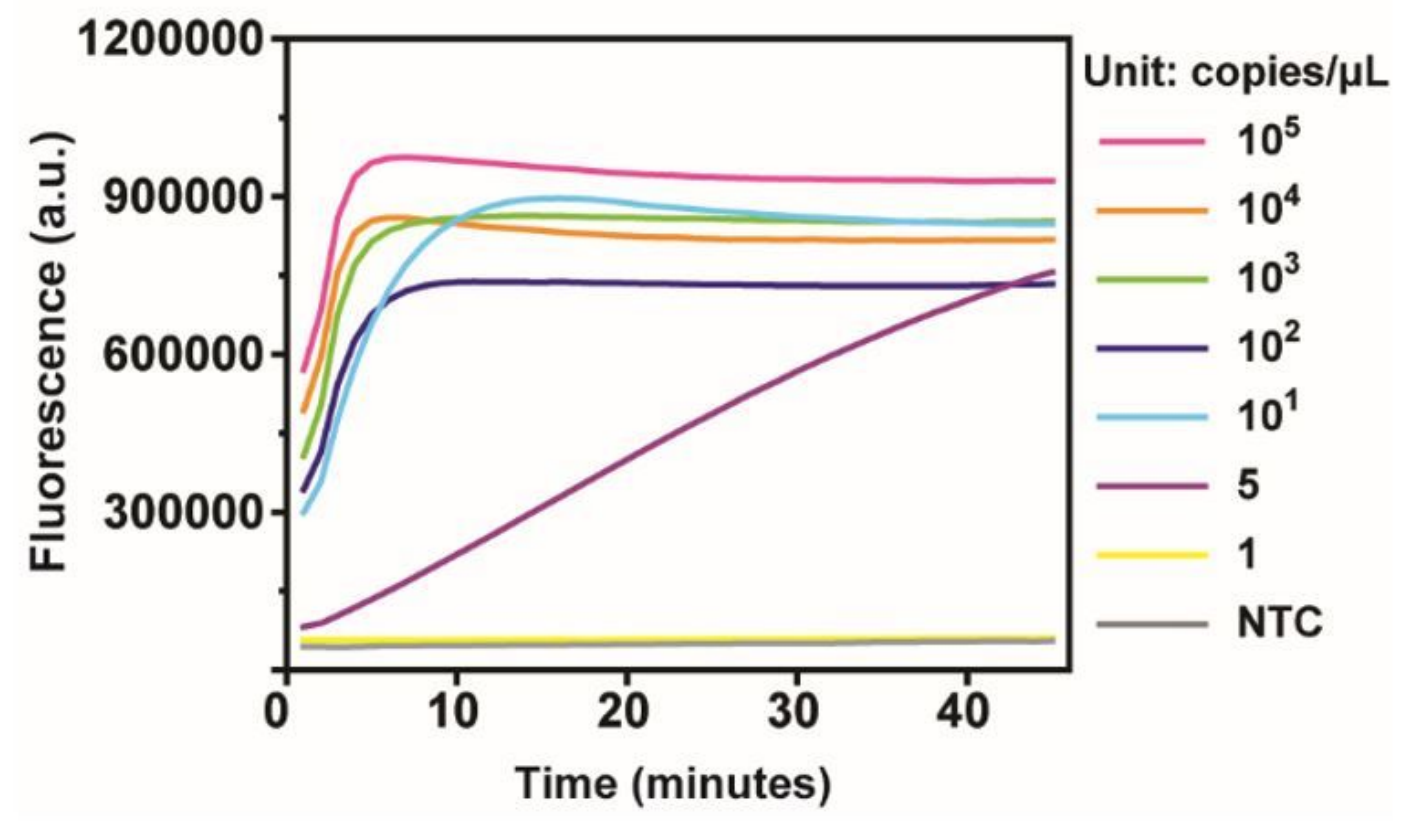

Figure 4 
Sensitivity of the CRISPR-GBS assay for GBS detection. A serial concentration of GBS plasmid DNA were used for detection of the fluorescence values. No template control (NTC), which targeted nucleic acid, was equally substituted by molecular grade water.

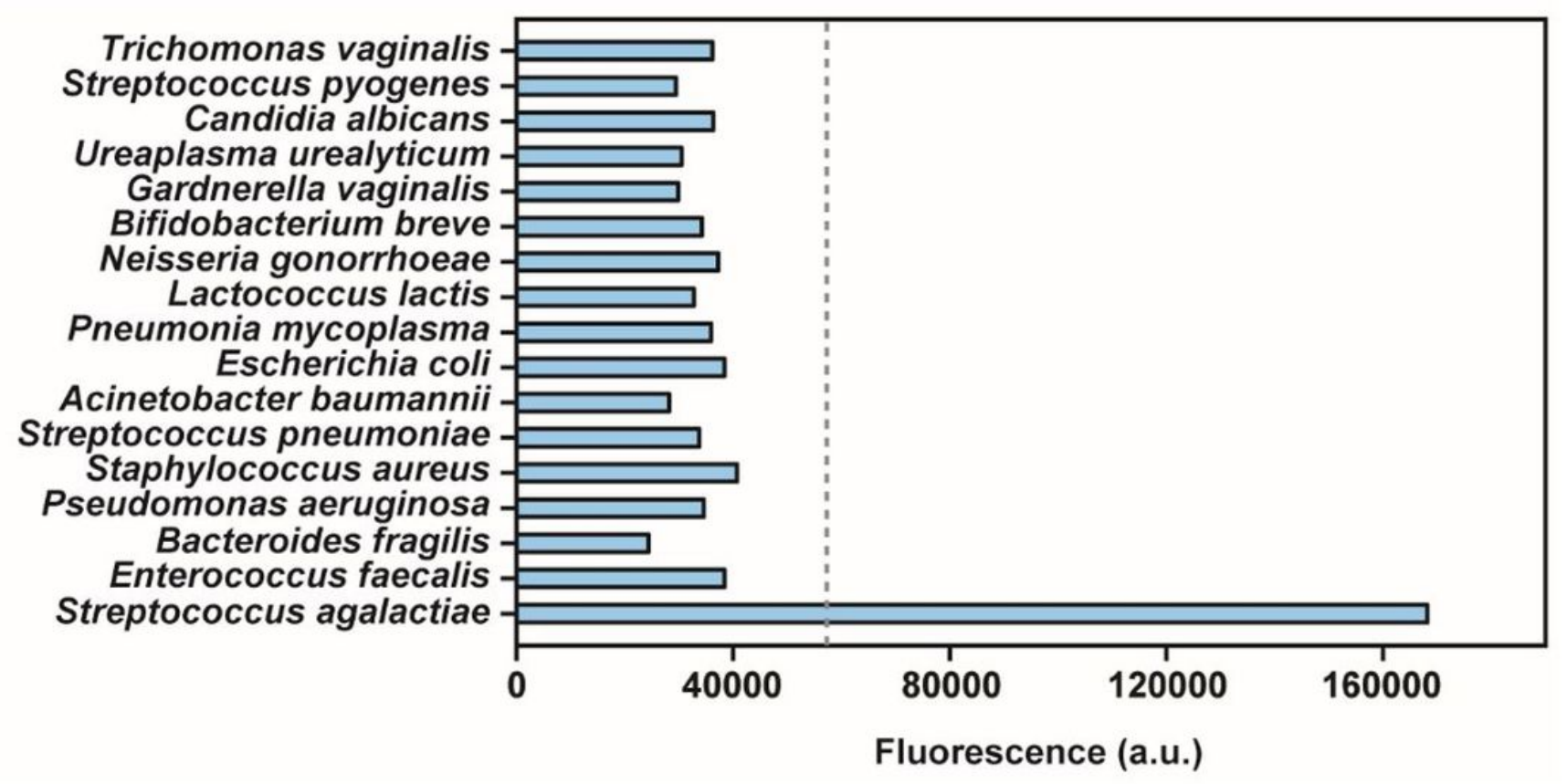

Figure 5

Specificity of the CRISPR-GBS assay for GBS detection. Specificity of the CRISPR-GBS assay for GBS detection was compared against other microorganisms in the vaginal/anal flora. The dashed line means cut-off value of CRISPR-GBS assay determined by the ROC curve (see Method: Determination of cut-off value for the CRISPR-GBS assay).

A

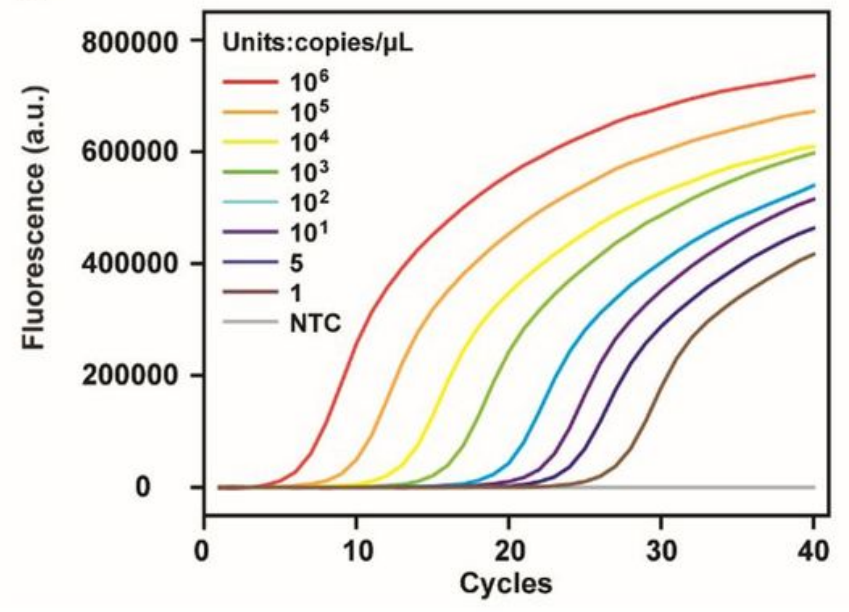

B

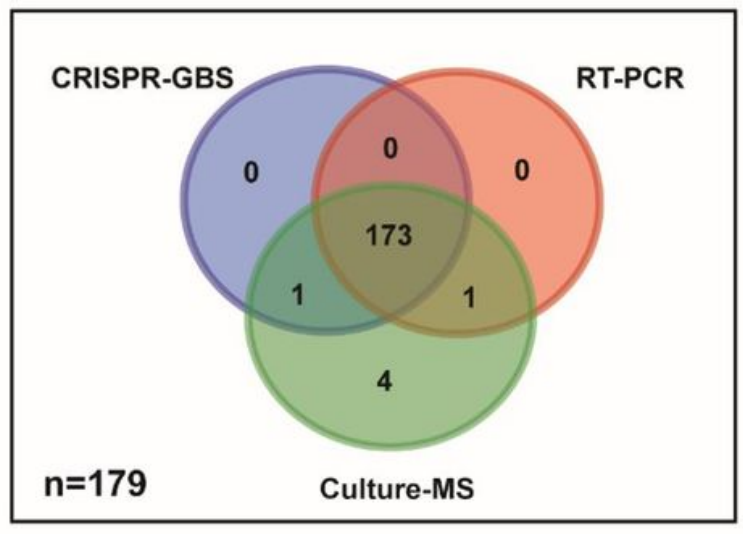


Figure 6

The performance of CRISPR-GBS assay on clinical samples detection. (A) The performance of RT-PCR detection method was validated by GBS plasmid DNA in the range of 1 to $106 \mathrm{copies} / \mu \mathrm{l}$. (B) Venn diagram shows the results of GBS detection by CRISPR-GBS (blue circle), RT-PCR (red circle) and cultureMS (green circle) assays using clinical samples $(n=179)$.

\section{Supplementary Files}

This is a list of supplementary files associated with this preprint. Click to download.

- Additionalfilesupload11.15.docx 